Palabras clave: Reacción post-traumática, Trauma de guerra, Psicosis de guerra, Neurosis de guerra, Trastorno de Estrés Post-traumático, Soldados de Naciones Unidas, Voluntariado.

\title{
Síntomas de Estrés Postraumático entre los soldados de Naciones Unidas y el personal perteneciente al voluntariado
}

\author{
Marianne Kaspersen Psicóloga* \\ Stig Berge Matthiesen Psicóloga** \\ * Universidad Noruega de la Ciencia y \\ Tecnología (NTNU), Trondheim \\ ** Universidad de Bergen \\ NORUEGA
}

RESUMEN - Objetivo: El artículo describe las reacciones postraumáticas entre dos grupos de individuos expuestos a la guerra, evaluándose el grado de asociación de con los traumas violentos.

Métodos: En la exposición al trauma entre los soldados de las Naciones Unidas y el personal del voluntariado se evaluó la sintomatología postraumática y la psicopatología general (únicamente ésta entre el voluntariado).

Resultados: Solamente del 2,8 al 7,8\% de los participantes puntuaron por encima del límite en cuanto a la sintomatología relativa al Trastorno de Estrés Post-traumático (PTSS, evitación-IES, intrusión-IES), con una excepción que fue que en la escala de intrusiónIES, el 20,8\% de los soldados de las Naciones Unidas se situaron por encima del límite. Entre el voluntariado, la violencia dirigida contra otros, se encontraba consistentemente asociada con la presencia de psicopatología, ambas generales y relacionadas con el trauma, mientras que la violencia dirigida contra uno mismo no se relacionaba con ningún tipo de psicopatología. Entre los soldados de las Naciones Unidas estas relaciones no tenían consistencia alguna.

Conclusiones: Las diferencias en cuanto al grado de exposición al trauma no pueden explicar los resultados, y son por tanto indicativos de la existencia de diferencias entre los dos grupos a lo largo de dimensiones que determinan la percepción del trauma, la personalidad o las estrategias de imitación. Se necesitan más estudios para establecer estas diferencias. 


\section{Introducción}

Las investigaciones acerca del Trastorno de Estrés Postraumático (PTSD) y de las situaciones de estrés relacionadas con acontecimientos traumáticos han mostrado dos tipos de hallazgos.

El primero, que los pacientes que cumplían con los criterios diagnósticos del Trastorno de Estrés Postraumático (PTSD) casi siempre cumplían también los criterios con otro trastorno o enfermedad psiquiátrica (modelo de comorbilidad). El segundo se refiere a que la predicción de un Trastorno de Estrés Postraumático a través de la sola experiencia traumática ha revelado que el criterio del estresor solamente supone una cierta cantidad de la varianza teniendo en cuenta el inicio y el mantenimiento del PTSD. La prevalencia del PTSD entre los veteranos de guerra varían desde un $2 \%$ en el Vietnam Experience Study, en el Centro de Control de Enfermedades (CDC 1988), hasta un $70 \%$ encontrados en otros estudios (Ver McFarlane \& Girolamo 1996, Davidson \& Fairbank 1993). Sin embargo, parece tambien existir una relación dosis-dependiente entre la exposición al trauma y la reacción propiamente traumática, sugiriendo ello la importancia que realmente tiene la propia exposición al trauma. Por otra parte, se ha sugerido que otros parámetros del trauma serían los responsables de algunas de las variaciones en cuanto a la sintomatología que la exposición al trauma puede producir. Uno de estos factores es la exposición a la violencia. Más específicamente, en el caso de ésta se ha tenido en cuenta un tipo de violencia dirigida contra los demás, de aquella otra que se dirige contra uno mismo, especificación ésta que también ha sido contemplada en cuanto a los criterios para el diagnóstico de PTSD según el DSM-IV.
La exposición al trauma guarda relación tanto con los rasgos subjetivos como con los objetivos. A pesar de que el tratar de los aspectos teóricos va más allá del marco de este estudio, hemos de señalar cómo varias de las características relevantes en las reacciones post-traumáticas, tales como la pérdida y falta de control, la impotencia, la amenaza, ciertas miradas y sonidos (a menudo grotescos) y la humillación, se conectan también con aspectos subjetivos y objetivos de la violencia en términos de violencia contra los demás y contra uno mismo. Los traumas producidos en las guerras a menudo conllevan la exposición a ambos tipos de violencia.

La violencia agresiva limita el diagnóstico de Trastorno por Estrés Post-traumático (PTSD) mediante el criterio de numeración y de evitación, siendo considerada como uno de los aspectos del trauma que determinan los criterios para un diagnóstico de PTSD (Breslau 2001). Sin embargo, en cuanto a este aspecto, existe diferencia entre hombres y mujeres. Breslau et al. (1999) descubrieron la existencia de una diferencia significativa entre hombres y mujeres con respecto a que el porcentaje se relacionaba con el criterio de numeración y el de evitación en el diagnóstico DSM-IV una vez habían sido expuestos a la violencia agresiva (autolesiva). Sin embargo no hay diferencia alguna entre hombres y mujeres en lo que respecta a los criterios de numeración y evitación, después que se hubiera percibido la violencia en contra de otras personas. Además, la diferencia existente en los hombres entre una violencia agresiva (violencia contra uno mismo) y traumática para con otros (violencia hacia otros) era insignificante si lo comparamos con la diferencia que existía entre las mujeres. Es de esperar que en una muestra compuesta en su mayor parte por hombres, el valor predictivo diferencial de la violencia autolesiva versus la dirigida contra los otros desapareciera prácticamente. 
Los individuos que habían sufrido exposiciones a situaciones traumáticas con anterioridad, poseían un mayor riesgo para el desarrollo de Trastorno de Estrés Postraumático (PTSD) que aquellos otros que no las habían experimentado. Esto tiene que ver especialmente con la exposición a la violencia y ha sido hallado en diferentes tipos de traumas (Breslau 2001) incluyendo a los veteranos de Vietnam (Kulka et al. 1990, Zaidi \& Foy 1994, Bremner et al. 1993). Basándonos en todo esto y en los descubrimientos mencionados anteriormente, podríamos imaginar que el grado y el tipo de exposición a la violencia, puede claramente considerarse como un buen predictor de sintomatología del PTSD, tanto de forma completa como parcial.

La exposición al trauma normalmente se halla relacionada con una cantidad limitada de la varianza total en lo referente a la sintomatología del Trastorno de Estrés Postraumático (PTSD). Esta variabilidad podría ser causada por efecto de la interacción entre la exposición al trauma, los factores sociales y otras diferentes características propias de la personalidad (modelo de la vulnerabilidad) o podría ser explicada a través de las variadas facetas de la exposición al trauma, dando lugar a diferentes tipos de síntomas (modelo de la comorbilidad) y a diferentes intensidades de los mismos. En el presente estudio, nosotros tenemos en cuenta el modelo propio de la comorbilidad. Snow et al. (1998) encontraron una prevalencia del PTSD entre los veteranos del Vietnam que variaba entre el $1,8 \%$ y el $15 \%$ de acuerdo con el nivel del estrés propio de cada zona de guerra. En los Centros de Control de Enfermedades, se encontró que un $15 \%$ de una muestra de 2.490 veteranos sufrían de un Trastorno de Estrés Postraumático (PTSD) relacionado con el combate. Sin embargo, la prevalencia un mes anterior a la valoración (PTSD actual) fue únicamente de un $2,2 \%$. Resulta interesante esta diferencia de resultados encontrados por el Estudio Nacional sobre la Rehabilitación de los Veteranos del Vietnam (NVVRS) que fue dirigido por Kulka et al. (1990). Descubrieron así como existía un predominio de Trastorno de Estrés Postraumático (PTSD) de un $15,2 \%$, en contraste con un $2,2 \%$ en el estudio realizado por los Centros para el Control de Enfermedades. Podría haber diferencias metodológicas que fueran en parte responsables de estos resultados, como suele ocurrir cuando los instrumentos de valoración son diferentes, sin embargo estos resultados requieren una más profunda investigación, preferiblemente teniendo en cuanta diferentes tipos de muestras con individuos expuestos a estos traumas.

Teniendo en cuenta la comobilidad, se han encontrado una gran variedad de trastornos presentes en las muestras de PTSD. Davidson et al. (1991) encontraron como los trastornos de somatización $(90,2 \%)$ y la esquizofrenia $(37,0 \%)$ eran los dos tipos de cuadros más frecuentes entre estos enfermos, mientras que Shore et al. (1986) hallaron especialmente trastornos de ansiedad generalizados $(76 \%)$ y depresiones $(51 \%)$. En este artículo nosotros estamos especialmente interesados en las dimensiones propias de la ansiedad, la depresión y las somatizaciones.

El propósito de este estudio es el de detectar y valorar el trauma relacionado con la psicopatología presente en dos grupos diferentes de personas que habían actuado en la antigua Yugoslavia. Estas son las cuestiones que el estudio trataba de responder: 1) ¿Cuántos individuos cumplirán con el criterio fijado para las reacciones postraumáticas de acuerdo con el Impacto de la Escala de Acontecimientos (IES) y la Escala de Estrés Post-traumático (PTSS) (Holen 
et al. 1983) en dos grupos diferentes de personas expuestas en una situación bélica? 2) ¿Existirá alguna diferencia en cuanto a la asociación estadística entre la exposición al trauma, definida como violencia dirigida contra uno mismo y violencia contra los demás, y diferentes tipos de patologías relacionadas y no relacionadas con el trauma? 3) ¿Existirán algunas diferencias entre los dos grupos en relación con todo ello? 4) ¿Habrá alguna diferencia entre la psicopatología medida con el SCL-90 y la relacionada con el trauma? Basándonos en otros resultados presentados con anterioridad, estamos interesados en los problemas de somatización, ansiedad y depresión como dimensiones patológicas generales.

\section{Métodos}

\section{Individuos}

Dos muestras son las que han participado en este estudio. Una compuesta por 302 voluntarios que participaron en el servicio de ayuda en Yugoslavia durante el periodo que va desde el año 1992 al 1996, y por otro lado una muestra compuesta por los observadores militares noruegos (Norwegian United Nations Military Observers) tratándose de soldados de las Naciones Unidas que sirvieron en la antigua Yugoslavia durante ese mismo periodo. A ambos grupos se les administró el mismo cuestionario, excepto a los soldados de Naciones Unidas que no recibieron el SCL-90. En la muestra de las Naciones Unidas se encontraban representadas todas las divisiones militares (fuerzas navales, de tierra y de aire). De entre los 97 soldados de las Naciones Unidas, 72 personas devolvieron los cuestionarios $(74,2 \%$ con un rango de edad comprendido entre los 32-54 años y con una media de 44,1 años).
La muestra de los voluntariados comprendía 141 individuos (46,7\% con edad comprendida entre los 26 y 66 años con una media de 42,8 años). La duración del servicio (para ambos grupos) se extendía de 1 a 37 meses con una media de 10,4 meses.

En la muestra del voluntariado el $85,1 \%$ eran hombres y en la de las Naciones Unidas, únicamente 9 personas $(12,5 \%)$ eran mujeres. Por tanto, el sexo no se trató como una variable en este estudio. Debido al bajo porcentaje de respuestas en la muestra del voluntariado, se llevó a cabo un análisis de frecuencias sobre las categorías de trabajo con el fin de asegurar que esta muestra no era parcial en ninguna dirección. Este análisis reveló que el $12,8 \%$ no trabajaba, y el resto de los participantes se distribuían entre seis categorías de trabajo de la siguiente manera: trabajadores de cuello azul 17,0\%, trabajadores de cuello azul profesional $18,4 \%$, trabajadores de cuello blanco $9,9 \%$, trabajadores de cuello blanco profesional $18,4 \%$, trabajadores en puestos de dirección: $19,1 \%$ y finalmente un $15,6 \%$ eran personas con ocupaciones independientes. A excepción de la categoría de los de cuello blanco, la muestra no parecía ser particularmente desviada en cuanto a las categorías de trabajo de las que se componía.

\section{Instrumentos}

La exposición al trauma (TE). La exposición al trauma se midió mediante un cuestionario compuesto de 8 preguntas. Este cuestionario se basó en uno que se utilizó en un estudio realizado en el Líbano, en las Fuerzas Interinas de las Naciones Unidas (UNIFIL) (Weisaeth 1993). El cuestionario recoge información sobre experiencias relacionadas con la guerra así como sobre testimonios vividos por el individuo. Consiste en cuatro cuestiones que tienen que ver sobre la vio- 
lencia traumática dirigida contra uno mismo (V/T-S) y otras cuatro que tratan sobre la violencia dirigida contra otros (V/T-O).

Estrés Post-traumático. El PTSS-10 (Holen et al. 1983) es una escala consistente en 10 items que miden los síntomas de estrés post-traumático de un determinado tipo. La medida es bastante fiable. El alfa de Cronbach era de 0,90 entre el voluntariado y de 0,84 en la muestra de los soldados de Naciones Unidas. La escala existe en dos versiones, una corta y otra estándar. En la versión estándar las respuestas van graduadas de cero a siete, mientras que en la versión corta, los encuestados solamente comprueban si los síntomas están o no presentes. La versión estándar de esta escala se utilizó entre el voluntariado y la versión corta entre los soldados de las Naciones Unidas. Con el fin de que ambos grupos fueran comparables, la escala de los síntomas se recodificó en una versión dicotomizada para el voluntariado. Los análisis de fiabilidad mostraron un alfa de Cronbach $=0,90$ entre el voluntariado (antes de la dicotomización) y de 0,84 entre los soldados de las Naciones Unidas. De acuerdo con Holen et al. (1983) una puntuación de 6 o mayor en la versión dicotomizada, podría indicar una disfunción patológica, por lo que se utilizó éste como punto de corte con el fin de evaluar la presencia de patología.

Escala de Impacto de Acontecimientos. El IES (Horowitz et al. 1979) es una escala compuesta por 15 cuestiones y diseñada para recoger acontecimientos subjetivos relacionados con el estrés. Consiste en dos subescalas que detectan los traumas relacionados con la sintomatología intrusiva y de evitación. Las medidas proporcionadas por la IES son de gran fiabilidad. En este sentido se recomienda como punto de corte para la evaluación de la intrusión y la evitación patológicas una puntuación de 20 o mayor tanto en cuanto a la intrusión IES como a la evitación IES. Pues bien, fue este el valor que se utilizó en este estudio.

Lista de chequeo de síntomas-90 (SCL 90). El SCL -90 (Derogatis 1977) es una lista compuesta por 90 cuestiones relativas a diversos tipos de síntomas. El SCL-90 comprende una medida general (El Índice de Gravedad General (GSI)), y 10 subescalas que miden procesos patológicos específicos. Las propiedades psicométricas del SCL-90 se consideran como buenas (Derogatis \& Clearly 1977). En este estudio se utilizaron las siguientes escalas: Somatización, Ansiedad, Ansiedad Fóbica y Depresión. El punto de corte se fijó como de 1,6 o superior.

\section{Resultados}

Los resultados revelaron que el PTSD relativo a la sintomatología teniendo en cuenta el punto de corte recomendado para el PTSS y para las escalas IES mostraba entre el voluntariado una prevalencia del 2,8 al 7,8\%. Entre los soldados de las Naciones Unidas, sin embargo la variación era mayor oscilando entre un 5,6\% y hasta un $20,8 \%$. Los síntomas de intrusión entre soldados de las Naciones Unidas eran bastante altos, cerca de tres veces superiores a lo que ocurría entre el voluntariado. (Ver tabla I).

La tabla II muestra que nuestra predicción en el sentido de que no habrían diferencias en cuanto a la asociación estadística entre la V/T-O y las medidas dependientes versus la V/T-S, no puede ser soportada con los datos actuales. Los resultados revelan también una diferencia entre los dos grupos. En el caso del voluntariado todas las correlaciones entre la V/T-O y las medidas dependientes son estadísticamente signifi- 
cativas, mientras que únicamente la evitación IES es estadísticamente significativa con respecto a la V/T-S. Por tanto se encontró una diferencia consistente entre la V/Y$\mathrm{O}$ y la V/T-S, entre los soldados de las Naciones Unidas (ver tabla II).

La tabla II muestra cómo la psicopatología no relacionada con los traumas (somatización, ansiedad, depresión y ansiedad fóbica) se halla fuertemente (y más fuertemente) relacionada con la V/T-O tal como lo está la psicopatología relativa al trauma. La V/T-S no se encontró que estuviese relacionada con ninguna de las medidas del SCL-90. Sin embargo, no había diferencia en cuanto a la fuerza de la asociación de la V/T-O entre las diferentes medidas del SCL-90.

La correlación entre la edad y la exposición al trauma no era significativa. Tampoco existía ninguna correlación significativa entre la edad y alguna de las variables del síntoma. Estas asociaciones estaban cerca de cero, por lo que no fue necesario investigar en lo referente a ningún grupo específico de edad.

Los soldados de las Naciones Unidas estuvieron significativamente más expuestos a los traumas que el personal voluntario $(p<, 001)$, y ello tanto en cuanto a lo referente a la violencia contra los otros como en cuanto a la violencia autolesiva.

Tabla I

Número de individuos con diagnóstico de Trastorno de Estrés Postraumático o de otra patología en porcentajes

\begin{tabular}{|c|c|c|c|c|}
\hline \multirow[t]{2}{*}{ Variable } & \multicolumn{2}{|c|}{$\mathrm{RW}(\mathrm{N}=141)$} & \multicolumn{2}{|c|}{$\mathrm{UN}(\mathrm{N}=72)$} \\
\hline & Total & $\%$ & Total & $\%$ \\
\hline PTSS $(\geq 6)$ & 4 & 2,8 & 4 & 5,6 \\
\hline IES-Intrusión ( $\geq 20$ ) & 11 & 7,8 & 15 & 20,8 \\
\hline IES-Evitación ( $\geq 20)$ & 5 & 3,5 & 4 & 5,6 \\
\hline SCL-Somatización $(\geq 1,6)$ & 3 & 2,1 & - & - \\
\hline SCL-Ansiedad $(\geq 1,6)$ & 3 & 2,1 & - & - \\
\hline SCL-Depresión $(\geq 1,6)$ & 4 & 2,8 & - & - \\
\hline SCL-Ansiedad fóbica $(\geq 1,6)$ & 1 &, 7 & - & - \\
\hline
\end{tabular}

$\mathrm{RW}=$ Voluntariado.

$\mathrm{UN}=$ Soldado de Naciones Unidas.

Tabla II

Correlaciones entre la exposición al trauma y las variables dependientes entre personal perteneciente al voluntariado y soldados de Naciones Unidas

\begin{tabular}{lccccccc} 
Variable & SOM & ANX & DEP & FOB & PTSS & IES I & IES A \\
\hline V/T S-RW &, 12 &, 26 &, 01 &, 14 &, 11 &, 08 &, $55^{* *}$ \\
V/T O-RW &, $36 * *$ &, $43 * *$ &, $32^{*}$ &, $47 * *$ &, $33^{*}$ &, $31^{*}$ &, $40^{*}$ \\
V/T S-UN & - & - & - & - &, 14 &, 17 &, $28^{*}$ \\
V/T O-UN & - & - & - & - &, 20 &, $28^{*}$ &, $33^{*}$ \\
\hline
\end{tabular}

$\mathrm{V} / \mathrm{T} \mathrm{S}=$ Violencia o amenazas contra sí mismo.

$\mathrm{V} / \mathrm{T} \mathrm{O}=$ Violencia o amenazas contra los demás.

SOM = Somatización. ANX= Ansiedad.

DEP = Depresión. FOB = Ansiedad fóbica. PTSS = Escala de Estrés Postraumático.

IES = Impacto del acontecimiento (puntuación total). IES I = Intrusión. IES A = Evitación. 


\section{Discusión}

Cuatro son los principales resultados obtenidos en el presente estudio. El primero, es que fueron relativamente unos pocos de los que participaron en esta investigación los que mostraron puntuaciones IES y PTSS, suficientemente altas como para ser considerados con patologías relacionadas con el trauma. Únicamente había una excepción, la del alto porcentaje de soldados de las Naciones Unidas que manifestaron síntomas intrusivos $(20,8 \%)$. El segundo resultado mostró como solamente la V/T-O estaba significativamente asociada con medidas dependientes entre el voluntariado. Además nuestra predicción acerca de que la diferencia en cuanto a la asociación mediante medidas dependientes entre la V/T-O y la V/T-S desaparecería en una muestra compuesta básicamente por hombres no tuvo el necesario soporte. En tercer lugar, los resultados revelaron también una diferencia entre los soldados de las Naciones Unidas y el voluntariado en cuanto a la asociación estadística existente entre exposición al trauma y patología relacionada con el PTSD. En cuarto lugar, no existía diferencia entre la patología psiquiátrica general medida con el SCL-90, y la patología relacionada con el trauma entre el voluntariado.

Un porcentaje relativamente bajo de informantes padecían de un PTSD en este estudio. Esto está de acuerdo con algunos otros estudios sobre la prevalencia del PTSD entre individuos expuestos a las situaciones de guerra (CDC 1988), pero aún esta prevalencia era más baja que la encontrada por Kulka et al. (1990). Se ha sugerido que si por ejemplo se emplea el DIS para diagnosticar un PTSD, tal como hicieron Kulka et al. (1990), solamente se pueden llegar a identificar un 25\% de los casos (ver Davidson \& Fairbank 1993). En el estudio de CDC se utilizaron muchas formas de medidas. Por tanto, es importante tener en cuenta que las diferencias metodológicas podrían complicar los intentos de hacer comparaciones entre diferentes estudios.

Nuestros datos revelan una excepción, la del alto porcentaje de soldados de las Naciones Unidas con síntomas intrusivos $(20,8 \%)$. Una explicación para esto podría ser que los soldados de las Naciones Unidas tuvieran mayores exposiciones a los traumas que el voluntariado. Sin embargo, debido al hecho de que el número de informantes que puntuaba por encima del límite era aproximadamente el mismo para ambos grupos en ambas escalas, la de IES-evitación y la de PTSS, la explicación no puede ser verdaderamente aceptable. Es más razonable asumir que los soldados de las Naciones Unidas poseen ese alto porcentaje de intrusión-IES debido a diferentes razones. Una posibilidad es que la escala de intrusión-IES no mida las reacciones postraumáticas en sus fases finales. El hecho de que algunos soldados de las Naciones Unidas se encuentren por encima del límite en la escala PTSS, podría, de acuerdo con Creamer et al. (1992), indicar que los soldados de las Naciones Unidas estén más capacitados para procesar la memoria traumática, pero disminuyendo las posibilidades para desarrollar un PTSD relacionado con la psicopatología. Estos procesos, podrían incluir varias maneras de coping con traumas, así como otras dimensiones de la personalidad.

Entre el voluntariado, la V/T-O estaba positivamente asociada a todas las escalas de síntomas, mientras que la V/T-S únicamente se asociaba a la escala de evitación. Entre los soldados de las Naciones Unidas esta diferencia no era muy consistente. Sin embargo, nuestra predicción acerca de que la diferencia entre la V/T-O y la V/T-S desapareciera en una muestra constituida principalmente por 
hombres, no recibió suficiente soporte. La escasez de los valores predictivos diferenciales de la V/T-O versus la V/T-S entre los hombres, encontrada por Breslau et al. (1999) no ha podido ser replicada por nuestra investigación. Hay que recordar que tuvimos solamente una medida de evitación y ninguna medida para la el bloqueo emocional. Sin embargo, lo que resulta interesante es que encontramos una V/T-O (violencia evidente) positivamente correlacionada con las medidas dependientes (IES-evitación, IES-intrusión y PTSS ) mientras Breslau et al. (1999) encontraron una violencia agresiva (T/V-S) asociada con la evitación y el bloqueo emocional. Nuestros resultados confirman la existencia de diferencias con respecto al sexo, y dichas diferencias parecen estar relacionadas con la V/I-S o la V/I-O que es el tipo de violencia asociada a la sintomatología.

Los resultados obtenidos en la muestra del voluntariado, podrían indicar que la exposición a un trauma visual se encuentra consistentemente más relacionada con la psicopatología que la violencia dirigida o experimentada como dirigida contra uno mismo. En la muestra del voluntariado la V/T-S se encuentra significativamente relacionada con la evitación-IES. Esto indica que la V/T-S podría hacer que la gente olvidara los recuerdos o disparos, pero no necesariamente se trata de un factor determinante en el desarrollo de sintomatologías intrusivas o de otras relacionadas con el Trastorno de Estrés Postraumático (PTSD).

Las diferencias existentes entre el voluntariado y los soldados de las Naciones Unidas, podría sugerir que ambos grupos estuvieron expuestos al trauma, pero de maneras diferentes. Los resultados revelan que los soldados de las Naciones Unidas se hallaron significativamente más expuestos a los traumas que los individuos del voluntariado, y ello en todos los items estudiados, tanto en los referentes a la V/T-S como en los de la $\mathrm{V} / \mathrm{T}-\mathrm{O}$. La razón por la que los items de la $\mathrm{V} / \mathrm{T}-\mathrm{O}$ se encuentran asociados a todas las medidas dependientes relacionadas con el trauma entre el voluntariado, mientras que esto no ocurre entre los soldados de las Naciones Unidas, podrían explicarse por otros factores que forman parte de la propia exposición al trauma. Las diferencias respecto a las estrategias de imitación, así como las referentes a las dimensiones de la personalidad, podrían ser factores a considerar, sin embargo las futuras investigaciones tendrán que determinar si es este el caso.

Las psicopatologías relacionadas y no relacionadas con el trauma, estaban significativamente asociadas a las medidas del trauma entre el voluntariado. Además, no había diferencia entre los varios tipos de psicopatologías (ansiedad, depresión y somatización) de acuerdo ello con la asociación estadística con la V/T-O. (Estas medidas no fueron válidas para los soldados de las Naciones Unidas). Esto podría hacer pensar que las reacciones psicopatológicas generales y las reacciones traumáticas específicas entre los voluntarios estén igualmente asociadas a la exposición al trauma, sugiriendo ello que la psicopatología general está más asociada a la exposición al trauma que lo que cabría esperar. Sin embargo, los resultados no apoyan la asociación de alguno de los grupos específicos de patología, con la patología relacionada con el Trastorno de Estrés Postraumático (PTSD). Por ello es más razonable sugerir que los actuales resultados van en concordancia con los otros hallazgos previos, los cuales señalan que las víctimas de estos traumas desarrollan una variedad de síntomas y de trastornos además de los del PTSD (ver McFarlane \& de Girolamo 1996). 


\section{Bibliografía}

BREMNER, J.D., SOUTHWICK, S.M., JOHNSON, D.R., et al. Childhood physical abuse and combat-related posttraumatic stress disorder in Vietnam veterans. American Journal of Psychiatry, 150, 235-239, 1993.

BRESLAU, N., CHILCOAT, H.D., KESSLER, R.C. et $a l$. Vulnerability to assaultive violence: on further specification of the sex difference in post-traumatic stress disorder. Psychological Medicine, 29, 813-821, 1999.

BRESLAU, N. The Epidemiology of Posttraumatic Stress Disorder: What Is the Extent of the Problem? Journal of Clinical Psychiatry, 62 (suppl. 17), 16-22, 2001.

CENTERS FOR DISEASE CONTROL (CDC): Health status of Vietnam veterans: psychosocial characteristics. JAMA, 259, 2701-2707, 1988.

CREAMER, M., BURGESS, P., PATTISON, P. Reaction to trauma: A cognitive processing model. Journal of Abnormal Psychology, 101, 452-459, 1992.

DAVIDSON, J.R.T., FAIRBANK, J.A. The Epidemiology of Posttraumatic Stress Disorder. In Davidson, J.RT. \& Foa, E.B. (eds.), Posttraumatic Stress Disorder: DSMIV and Bevond (pp. 37-54). Washington DC: American Psychiatric Press, Inc, 1993.

DEROGATIS, L.R. SCL-90-R Manual - I. Baltimore: Clinical Psychometric Research, 1977.

DEROGATIS, L.R., CLEARLY, P. A confirmation of the dimensional structure of the SCL-90: a study in construct validity. Journal of Clinical Psychology, 33, 981-989, 1977.

HOLEN, A., SUND, A., WEISAETH, L. PTSS-10: Questionnaire for screening disaster victims, in the Alexander Kielland disaster. March 27. 1980: Psychological reactions among the survivors, Oslo University, Division of Disaster Psychiatry (in Norway), 1983.
HOROWITZ, H.M., WILNER, N., ÁlVAREZ, W. Impact of Event Scale: A measure of subjective stress. Psychosomatic Medicine, 41, 209-218, 1979.

KULKA, R.A., SCHLENGER, W.E., FAIRBANK, J.A. et al. Trauma and the Vietnam War Generation: Report of Findings from the National Vietnam Veterans Readjustment Study, Branner/Mazel, NY, 1990.

McFARLANE, A.C., DE GIROLAMO, G. The Nature of Traumatic Stressors and the Epidemiology of Posttraumatic Stressors. In van der Kolk, McFarlane, A.C., and Weisaeth, L. (eds.): Traumatic Stress. The Effects of overwhelming Experience on Mind, body, and Society. The Guilford Press, NY, 1996.

SHORE, J.H., TATUM, E., VOLLMER, W.M. Psychiatric reactions to disaster. The Mt. St. Helen's experience. American Journal of Psychiatry, 143, 590-595, 1986.

SNOW, B.R., STELLMAN, J.M., STELLMAN, S.D. et al. Post-traumatic stress disorder among American Legionnaires in relation to combat experience in Vietnam: associated and contributory factors. Environmental Research, 47, 175-192, 1998.

WEISAETH, L. Forsvarets Sanitet. UNIFIL-Unders $\varnothing$ kelsen. 1991-1992. Rapport, Del I - 25.02.93. Resultater og anbefalinger, 1993.

ZAIDI, L.Y., FOY, D.W. Childhood abuse experiences and combat-related PTSD. Journal of Traumatic Stress, 7, 33-42, 1994.

Dirección para correspondencia:

Marianne Kaspersen

Norwegian University of Science and Technology (NTNU) Trondheim e-mail: marianne.kaspersen@medisin.ntnu.no NORUEGA 